Cartagena, 7 a 9 de septiembre de 2011

\title{
Modelo para la aplicación de la Responsabilidad Social Corporativa en la Gestión de la Cadena de Suministro
}

\section{A model for the Corporate Social Responsibility adoption in the Supply Chain Management}

\author{
Ana Mejías Sacaluga, Jesús García Arca, J. Carlos Prado Prado, Arturo J. Fernández González \\ y José A. Comesaña Benavides \\ Grupo de Ingeniería de Organización (GIO); Dpto. de Organización de Empresas y Marketing. \\ Escuela de Ingeniería Industrial de Vigo. \\ Universidad de Vigo. Campus Lagoas-Marcosende, C/ Maxwell, 363I 0.Vigo. \\ mejias@uvigo.es jgarca@uvigo.es jcprado@uvigo.es ajfdez@uvigo.escomesana@uvigo.es
}

Fecha de recepción: 7-9-2011

Fecha de aceptación: 19-9-2011

Resumen: En este trabajo se hace una revisión de la literatura sobre Responsabilidad Social Corporativa (CSR), especialmente la que contempla su relación con la logística y la cadena de suministro. Además, se analizan tres modelos recientes desarrollados bajo el concepto de Gestión Sostenible de la Cadena de Suministro. El objetivo de este trabajo es definir un nuevo modelo que complemente los anteriores con una perspectiva más aplicada o de despliegue hacia toda la organización. De este modo, se pretende que las empresas puedan aprovechar sus actuales planteamientos estratégicos, de gestión y operativos y reenfocarlos o alinearlos con la CSR.

Palabras clave: responsabilidad social corporativa, sostenibilidad, gestión de la cadena de suministro.

Abstract: In this paper a literature review about Corporate Social Responsibility (CSR) is presented, especially focused on its relationship with supply chain management (SCM). In this context, three recent models developing the concept of Sustainable Supply Chain Management (SSCM) are analysed. Going beyond, the main objective of this paper is to propose a new model that complements the previous ones, in the relationship of CSR and SCM, but with a more practical approach oriented to its deployment throughout the whole organization. Thus, thanks to this model, companies could consider their strategic, managerial and operative problems and realign them with CSR issues.

Keywords: corporate social responsibility, sustainability, supplies chain management.

\section{Introducción}

En esta última década ha aumentado el interés, tanto en el ámbito académico como en el empresarial, por la Responsabilidad Social Corporativa o Corporate Social Responsibility (en adelante, CSR), también llamada Responsabilidad Social Empresarial. Sin embargo, el concepto de CSR tiene una amplia trayectoria, cuyo tratamiento en la literatura de manera formal se remonta a la década de los 50 .

Por otra parte, es en la década de los 70 cuando las organizaciones intergubernamentales comienzan a posicionarse e involucrarse en los temas concer- nientes a la CSR. En 1976 la OECD (Organization for Economic Co-operation and Development) publica «Líneas directrices de la OECD para empresas multinacionales». En 1977, la OIT (Organización Internacional del Trabajo) publica la «Declaración tripartita de principios sobre las empresas multinacionales y la política social».Y una década más tarde, en 1987, la Comisión Mundial sobre Medio Ambiente y Desarrollo, promovida por Naciones Unidas, elabora un informe titulado «Our Common Future», más conocido como Informe Brundtland, en referencia al presidente de la comisión. En el informe se define por primera vez el término Desarrollo Sostenible como «La capacidad de satisfacer las necesidades de las gene- 
raciones presentes sin comprometer las posibilidades de las del futuro para atender sus propias necesidades»».

En el terreno práctico, se ha producido también un incremento notable de iniciativas empresariales en este campo; las empresas más destacadas han comenzado a formalizar sus prácticas en materia de CSR (memorias de sostenibilidad, mejores prácticas, etc.) y a difundirlas entre los grupos de interés; numerosas asociaciones y fundaciones diseñan modelos y guías de implantación de la CSR en las empresas; también, son diversas las normas internacionales para alcanzar certificaciones en este campo (AAI000, SA 8000,...).

Con todo, se considera que las implicaciones tan amplias que la CSR tiene para las organizaciones hace necesario profundizar en el estudio de sus diferentes ámbitos de gestión, en especial, la Gestión de la Cadena de Suministro o Supply Chain Management (en adelante, SCM), dada la importancia que ésta tiene en la competitividad de las empresas y sus impactos económicos, sociales y medioambientales. En este trabajo se presenta un marco de aplicación de la CSR a la SCM bajo una perspectiva completa (toda la cadena) y considerando todos los niveles de gestión (estratégico, táctico y operativo).

\section{CSR: estado del arte y necesidades de investigación}

En la revisión del estado del arte en la literatura sobre el tratamiento conceptual y empírico de la CSR destacamos dos amplios y profundos trabajos de recopilación. Por una parte, Carroll (1999), en su artículo «Corporate Social Responsibility. Evolution of a Definitional Construct», recoge de manera exhaustiva la evolución del concepto CSR desde la década de los 50, considerada «la era moderna» con respecto a la investigación en materia de CSR a raíz de la publicación del libro de Howard Bowen en 1953 «Social Responsibilities of the Businessman» (y que le ha servido el apelativo de «padre de la CSR»), hasta finales de la década de los 90. Con perspectiva de futuro, Carroll considera que si bien las investigaciones teóricas todavía tienen amplias posibilidades, será el complemento de los trabajos empíricos lo que permitirá un desarrollo completo y enriquecedor en este campo.

En segundo lugar, se debe citar el trabajo de Lee (2008), «A Review of the Theories of Corporate Social Responsibility: Its Evolutionary Path and the Road Ahe- ad», que supone un interesante complemento al trabajo de Carroll, además de ampliar el horizonte temporal a lo más reciente. Lee describe la evolución del concepto de CSR en las últimas tres décadas como de «racionalización» en dos sentidos. Primero, en lo que respecta al nivel de análisis, las investigaciones han evolucionado de la discusión o enfoque sobre los efectos macro-sociales de la CSR hacia el enfoque más organizativo del análisis de los efectos de la CSR sobre el beneficio. Segundo, en la orientación teórica, las investigaciones han evolucionado desde la visión de la normativa explícita y los argumentos basados en la ética, hacia la visión de la normativa implícita y los estudios basados en el desempeño en la gestión empresarial.

En su retrospectiva, el autor marca cuatro etapas en consonancia con Carroll. Los 50 y 60 o «Responsabilidades Sociales de los Hombres de Negocio» haciendo referencia al libro de Bowen, con un marcado carácter macro-social y ético. Los 70 o «Inspiración de Progreso», que supone más una corriente de pensamiento que un modelo teórico propiamente y que inicia la conciliación entre el interés social y económico de las empresas; no obstante, los conceptos de CSR y CFP (Corporate Financial Performance, Desempeño Financiero Corporativo) siguen separados y manteniendo su identidad.

En los 80, con el «Modelo de Desempeño Social Corporativo», llega el marco teórico conceptual esperado para la conciliación entre CSR y CFP con la publicación en 1979 en Academy of Management Review del trabajo de Carroll «A Three-Dimensional Conceptual Model of Corporate Performances; la mayor aportación de este modelo es que no trata los objetivos económicos y sociales de las empresas como trade-offs incompatibles, al contrario, ambos objetivos empresariales son integrados en un marco de responsabilidad social total de los negocios que incluye categorías económicas, legales, éticas y discrecionales (voluntarias). Posteriormente, Wartick y Cochan (1985) amplían el modelo de Carroll incorporando lo que denominan las dimensiones de principios, procesos y políticas (o aspectos de gestión). Con todo, según Wood y Jones (1995), este modelo denominado Corporate Social Performance (CSP) no consiguió un grado aceptable de aplicación, dado que carecía de un aspecto crítico de implantación: la capacidad para medir y testar empíricamente el modelo.

Finalmente, en la década de los 90, la «Gestión Estratégica» supone la aplicación del análisis relativo a los stakeholders (grupos de interés) a la investiga- 
ción sobre CSR. Así, frente a la falta de aplicabilidad de los modelos anteriores, el modelo stakeholders define las posiciones y funciones de todos estos agentes y las relaciones entre unos y otros, con lo que facilita el que el modelo se pueda probar o testar empíricamente. Esta nueva corriente de pensamiento tiene un gran desarrollo en la época más reciente: la adopción de la llamada «Filantropía Estratégica» ofrece nuevas oportunidades para la innovación, abre mercados y desarrolla valiosas relaciones sociales que pueden contribuir a la mejora de la reputación de la empresa (Porter y Kramer, 2002); Hart (1997) afirma que, considerando los enormes cambios medioambientales que se están experimentando, si el concepto de sostenibilidad se liga a la estrategia y al desarrollo tecnológico, las empresas pueden alcanzar ventajas competitivas considerables. Además, según Porter y Kramer (2006), en la CSR estratégica el concepto se aplica a todas las actividades del negocio así como al contexto competitivo de la compañía.

Tras esta retrospectiva, Lee señala que la racionalización del concepto de CSR y la convergencia entre CSR y el desempeño corporativo ha ido suscitando el interés en el tema entre los responsables de las compañías a todos los niveles. No obstante, el autor dedica la última parte de su trabajo a señalar las carencias de la investigación desarrollada hasta ahora en este campo y a proponer futuras líneas de investigación. En este sentido, el autor apunta varias oportunidades o líneas de investigación a reforzar. Por una parte, si bien la CSR y la CFP se han acoplado a los largo de la décadas, es escasa la verificación empírica de la relación entre la CSR y su influencia en los resultados empresariales (la mayor parte de la investigación empírica se enfoca en conocer el por qué y cómo las empresas implantan CSR). También indica que es preciso seguir desarrollando indicadores de medida que contribuyan a verificar las actuaciones que las empresas digan llevar a cabo. Por otra parte, los aspectos organizativos y la influencia que la CSR tiene en los procesos de toma de decisiones de los gestores tampoco están siendo tratados de manera suficiente. Asimismo, es importante profundizar en la dimensión social de la CSR, no sólo desde la perspectiva de la responsabilidad social de las empresas sino también, de manera bidireccional, de la respuesta de la sociedad hacia las empresas «socialmente responsables». Finalmente, el autor considera que los esfuerzos de investigación deben dirigirse a las pequeñas y medianas empresas, dada la importancia de su contribución al desarrollo económico y social de sus ámbitos de actuación.

\section{Logistics Social Responsability (LSR) y Sustainable Supply Chain Management (SSCM)}

En la mayor parte de los documentos, modelos o guías relativos a la CSR se menciona la necesidad de que las empresas entiendan su aplicación en el contexto más amplio de sostenibilidad, abarcando así todo lo referente a la cadena de suministro (en adelante, SC) (p.e. Comisión de las Comunidades Europeas, 200I; GRI, 2006; OECD, 2008, ISO 26000, 20I0).

Desde una perspectiva global o general, es lógico entender que la implantación de la CSR en las empresas no puede ser ajena a las estrategias y operaciones desarrolladas en el contexto de la SCM, ya sea por la propia definición de dimensiones interna y externa con la que las empresas deben enfocar su CSR, o por el diálogo/actuaciones que deben llevarse a cabo con las partes interesadas («stakeholders»), entre ellas, proveedores, subcontratistas, competidores y clientes.

No obstante, la revisión de la literatura sobre el estudio de las implicaciones de la CSR en la SCM o, de manera equivalente, en la gestión integrada de la logística, abarca sólo un período bastante reciente y además, en muchos casos, muestra un tratamiento parcial del tema, dado que se estudian sólo ámbitos concretos dentro de la cadena. Así, las investigaciones se han centrado, por ejemplo, en los aspectos medioambientales de la logística o la SCM, la seguridad en las operaciones de almacenaje y transporte - las cuestiones éticas de las relaciones ente fabricantes y proveedores.

Carter y Jennings (2002) presentan una revisión bibliográfica que recoge esta visión fragmentada de la logística en lo relativo a la CSR en los campos más habituales: medio ambiente, seguridad, ética e inclusión o diversidad. De manera sintética destacamos de la revisión que hacen los autores, en cuestiones medioambientales: logística inversa (Stock, 1998, 1992), SCM y el medio ambiente (Handfield et al. 1997), transporte de mercancías peligrosas (Sharp et al., 1991) y reducción del consumo de combustible y de las emisiones (McKinnon, et al., 1993). En cuestiones éticas, principalmente, se han estudiado las relaciones entre compras y proveedores de la industria de automoción y también de otros sectores industriales (Carter, 2000a, 2000b). En temas de seguridad, destacan los estudios en la industria automovilística y aeronáutica (Weener y Wheeler 1992) y de mejora de la ergonomía en el almacén (Randall, 1998). Por últi- 
mo, en cuestiones de inclusión, diversidad, igualdad, etc., destacan estudios acerca del nivel de profesionales femeninas en logística (Cooper and Gillyard, 2000), las compras procedentes de pequeños proveedores (Carter et al., 1999) o el respeto a los derechos humanos en plantas de proveedores de terceros países (Emmelhainz y Adams, 1999).

Más recientemente, se debe citar el trabajo de Porter y Kramer (2006), el cual responde al enfoque señalado por Lee (2008) como necesario en las investigaciones a llevar a cabo en la CSR, esto es, el involucramiento corporativo en la sociedad como enfoque estratégico. Para los autores, la integración de los negocios y la sociedad debe contemplar una doble dimensión: la vista de adentro hacia afuera, o trazar el impacto social de la cadena de valor, y la vista de afuera hacia adentro, o las influencias sociales sobre la competitividad de las empresas. De manera más precisa, los autores señalan que el impacto social de la cadena de valor puede tener un primer nivel, o de CSR reactiva, que implica mitigar el daño causado por las actividades de la cadena de valor, y un segundo nivel, o de CSR estratégica, que supone transformar las actividades de la cadena de valor para beneficio de la sociedad, reforzando la estrategia.

De este modo, la propuesta del impacto social positivo y negativo que todas las actividades de la clásica cadena de valor de Porter (1980) plantea un marco global en el que se ven reflejadas conjuntamente las actividades que abarca la gestión integrada de la logística en la empresa (abastecimiento, logística de entrada, operaciones, logística de salida y servicio postventa). En este contexto, Porter y Kramer (2006) plantean como evidente las actuaciones a llevar a cabo «...para la mayoría de los efectos de cadena de valor no hay necesidad de reinventar la rueda. La empresa debería identificar las mejores prácticas para lidiar con cada uno....».

No obstante, si bien el enfoque a seguir está claro para estos autores, coincidimos con Carter y Jennings (2002) en que, en el ámbito de la logística, todavía no se han agrupado todos los aspectos bajo un marco o paraguas que permita responder a la cuestión de cómo una empresa puede ubicar de manera efectiva el papel global de la logística en la CSR; precisamente, a través de la revisión de la literatura que llevan a cabo, la cual se ha citado anteriormente, se refleja la ausencia del marco o paraguas para crear una cultura de responsabilidad o un comportamiento que incremente la posición competitiva de la empresa a través de la mejora de las relaciones con los stakeholders (proveedores, suministradores, clientes...).
Tras la revisión bibliográfica, el trabajo de Carter y Jennings desarrolla una investigación empírica basada en entrevistas personales a 26 directivos de tres áreas funcionales de la logística, compras, almacenaje y transporte, con el triple objetivo de: identificar las actividades específicas que contempla la aplicación de la RSC al ámbito de la logística, y que los autores denominan Logistics Social Responsability (LSR), determinar las motivaciones o facilitadores y barreras para la LSR y determinar los resultados o consecuencias de aplicar la LSR, todo ello bajo un enfoque marcadamente cualitativo. Consideramos este trabajo un interesante punto de apoyo para la investigación que proponemos dado que, si bien el carácter exploratorio de la investigación de Carter y Jennings requiere la validación desde una perspectiva más cuantitativa, no obstante, ofrece un marco de referencia para el estudio de la aplicación de la CSR a la gestión integrada de la logística y, por tanto, a la SCM. Con todo, desde nuestra perspectiva, consideramos que se debería incluir en el estudio las áreas de producción y logística inversa y el propio diseño de la SC.

Además, entre las líneas de investigación a desarrollar que los autores señalan como recomendables, vuelven a mencionarse los aspectos organizativos, como ya se ha hecho referencia en la revisión del estado del arte de la CSR en general. A este respecto, consideramos dos cuestiones especialmente relevantes. Por una parte, los resultados exploratorios muestran que los gestores de logística (o de áreas funcionales), con su actitud, pueden influenciar positivamente en el desarrollo de la LSR dando forma a una cultura organizacional orientada a la CSR; de hecho, los resultados muestran que aunque una organización carezca de una cultura organizacional orientada hacia CSR, las iniciativas individuales de los gestores de logística, en base a su valores personales, pueden derivar en la implantación exitosa de programas y actividades de LSR. Por otra parte, la perspectiva del resto de agentes de la SC es necesaria, dado que una de las barreras más comunes en la LSR es la coordinación de objetivos y actividades entre las áreas funcionales internas (integración de la logística interna) y entre los diferentes agentes de la SC.

Tras la publicación del trabajo de Carter y Jennings (2002), la literatura más reciente muestra ya un enfoque más global hacia la relación entre CSR y la SCM. Aún así, son muchos también los trabajos que sólo analizan ámbitos concretos de la SC tratados de manera independiente. A modo de ejemplo, se pueden citar: gestión medioambiental (Handfield et al., 2005); suministro «verde» o ecológico (Sarkis, 2003; Vachon 
y Klassen, 2006; Mollenkopf et al., 20 I 0); y ética en la gestión del suministro (Eltantawy et al., 2009).

Pero, finalmente, conceptos como cadena de suministro sostenible (SSC), suministro ecológico o CSR en la SC implican ir más allá del enfoque de eficiencia puramente económica en el contexto competitivo actual. Hacer ecológica la SC puede salvar recursos, eliminar o reducir los residuos, y mejorar la productividad y las ventajas competitivas al mismo tiempo (Porter y Linde, 1995). Así, respondiendo a un enfoque más global, recientemente, se han publicado tres trabajos en los que se aborda una amplia revisión sobre la gestión sostenible de la SC (Carter y Rogers, 2008; Seuring y Müller, 2008, Pagell y Wu, 2009). En todos ellos se sugieren más o menos definiciones similares del concepto: «la gestión sostenible de la SC es la gestión de los flujos de material, información y capital, así como de la cooperación entre compañías a lo largo de la cadena que buscan lograr objetivos en las tres dimensiones del desarrollo sostenible, económica, medioambiental y social, teniendo en cuenta los requerimientos de los clientes y los demás stakeholders» (Seuring and Müller, 2008). De acuerdo con estos autores, la SSC está en total consonancia con la definición de desarrollo sostenible emanada del Informe Brundland (WCED, 1987) y que enfatiza la interdependencia ente los parámetros social, económico y medioambiental desde una perspectiva intergeneracional.

Esta perspectiva también se corresponde con la idea de la «triple cuenta», un concepto desarrollado por Elkington $(1998,2004)$, que simultáneamente considera y equilibra objetivos económicos, sociales y medioambientales bajo una perspectiva microeconómica. Así, la triple cuenta sugiere que en la intersección del desempeño económico, social y medioambiental, hay actividades en las que las organizaciones pueden tomar parte y en las que no sólo se afecta positivamente al entorno natural y a la sociedad, sino que también pueden proporcionar beneficios económicos a largo plazo y una ventaja competitiva para una empresa (Carter y Rogers, 2008).

\section{Modelos para la SSCM}

En este epígrafe se resumen las principales características de los tres modelos que se han desarrollado bajo el concepto de Gestión Sostenible de la Cadena de Suministro (SSCM), como paso previo a la definición del enfoque de este artículo, el cual, no pretende cuestionar la validez de estos modelos, si no que, por el contrario, busca complementarlos con una perspectiva más aplicada o de despliegue de los conceptos planteados hacia toda la organización. De esta manera, se busca que las empresas puedan aprovechar sus actuales planteamientos estratégicos, de gestión y operativos y reenfocarlos o alinearlos con la CSR.

\section{I. El modelo de Carter y Rogers}

Carter y Rogers (2008) proponen un marco para la SSCM basado en la triple cuenta, definiendo la SSCM como «el logro de los objetivos sociales, ambientales y económicos de una organización, a través de la integración estratégica, transparente y la coordinación sistémica de los procesos clave de negocios, entre organizaciones, para la mejora del rendimiento económico a largo plazo de la empresa y de su SC». Además, estas actividades deberían desarrollarse según las cuatro facetas que dan soporte a la triple cuenta: la estrategia, la gestión del riesgo, la cultura de la organización y la transparencia. En la Figura I se muestra el modelo.

Figura I

Gestión sostenible de la cadena de suministro. Fuente: Carter y Rogers (2008)

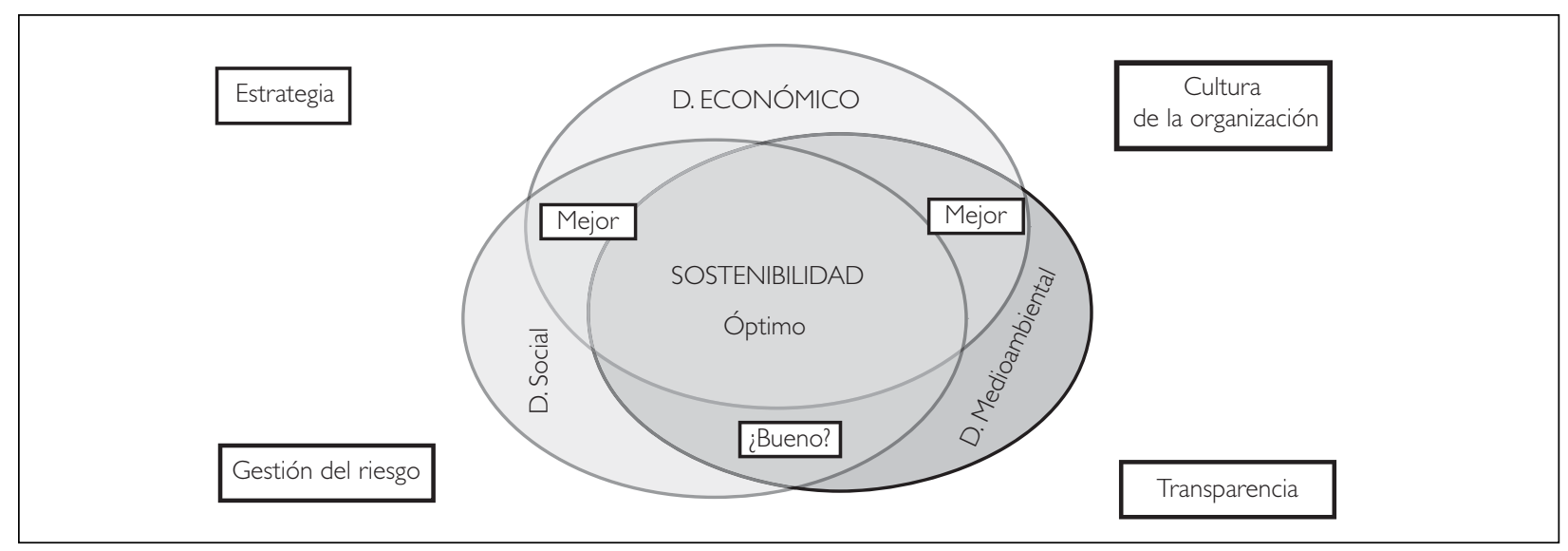

Dirección y Organización, Núm. 45, diciembre 201 I | ISSN (On line): 2 I7 I-6323 - ISSN (Print): II 32- I75X 
Teniendo esto en cuenta, los autores consideran que las actividades, tanto ambientales como sociales, que debe desarrollar o adoptar una empresa a lo largo de su SC, son aquellas que le permitan conseguir los objetivos económicos. En este sentido, los autores sugieren que las organizaciones no deberían centrarse solamente en cumplir los objetivos sociales y ambientales relacionados con la SC; de hacerlo así, coincidiendo con Porter y Kramer (2002), estas empresas serían socialmente irresponsables, a menos que esas acciones estén consideradas dentro del contexto más amplio de los objetivos estratégicos generales y financieros de la empresa. Los signos de interrogación en torno al término «bueno» hacen referencia a este aspecto, dado que en esa área del modelo tiene en cuenta las acciones sociales y medioambientales $y$, sin embargo, no se tiene en cuenta el aspecto económico. El modelo muestra, en cambio, que es «mejor» realizar actividades que permitan conseguir objetivos sociales o medioambientales separadamente, pero que tengan en cuenta el componente económico.

Finalmente, la verdadera sostenibilidad se consigue al ser capaz de aunar los tres componentes, económico, social y medioambiental. A este respecto, los autores recogen a través de una profunda revisión bibliográfica un conjunto de actividades enmarcadas en el área central de la sostenibilidad, en muchas de las cuales se busca la reducción de costes a través de actuaciones positivas en el ámbito social y medioambiental.

Por último, como todo marco conceptual, los autores consideran su modelo como un punto de parti- da a través de cual se siga investigando en líneas como: las creencias y motivaciones de las compañías para introducirse en la SSCM; la relación entre la cultura, estrategia, gestión del riesgo y transparencia en la SSCM; el empleo del estudio de casos para evaluar el propio marco teórico o la realización de un estudio longitudinal para evaluar el desarrollo económico a largo plazo.

\subsection{El modelo de Seuring y Müller}

En su modelo, Seuring y Müller (2008) tienen en cuenta también las tres dimensiones de la sostenibilidad y a todos los agentes que forman la SC. Los autores proponen un marco de referencia sobre el que poder crear SSCM, el cual se basa en tres puntos:

- Factores desencadenantes o facilitadores para la SSCM

- Gestión del riesgo y desempeño de los proveedores

- SCM para productos sostenibles

En el primer punto, los factores desencadenantes, se identifican a través de la literatura cuáles son las presiones externas y los factores que incentivan a las empresas a gestionar la SC de forma sostenible, así como los grupos de presión que actúan sobre las mismas. En la Figura 2 se muestra de manera gráfica los factores facilitadores para la SSCM, incluyendo también su relación con los otros dos puntos, gestión del riesgo y SCM para productos sostenibles.

Figura 2

Facilitadores para la SSCM. Fuente: Seuring y Müller (2008)

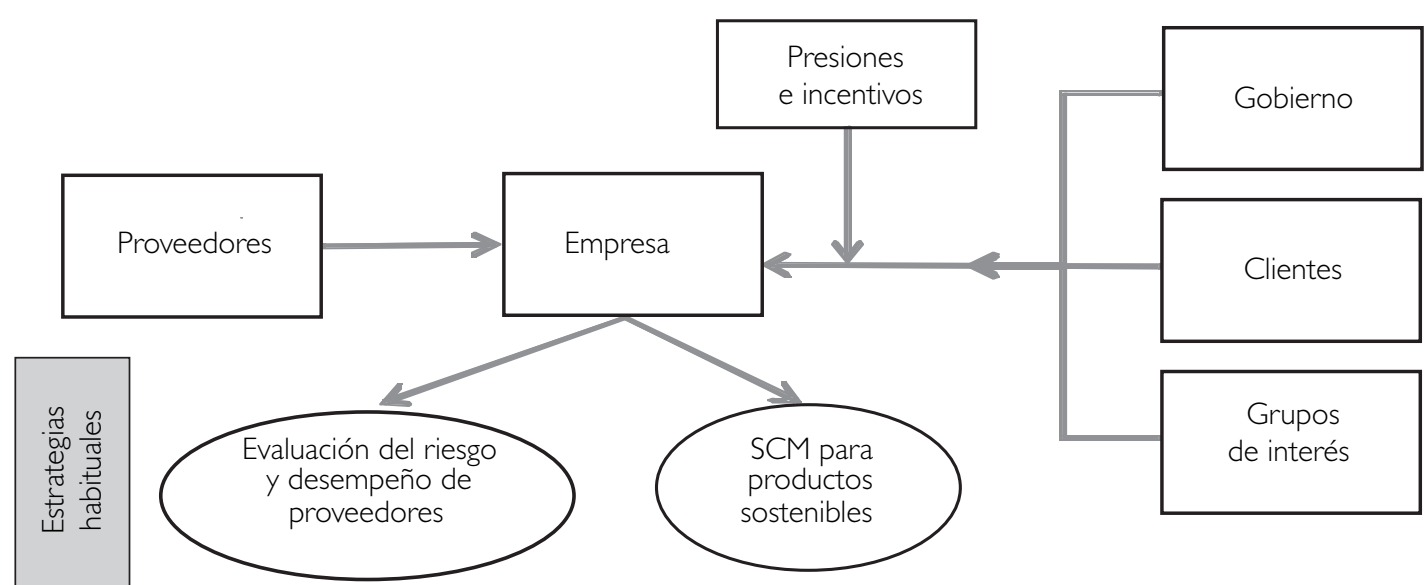


En cuanto a cuáles son estas presiones e incentivos que actúan como facilitadores de la SSCM, los autores señalan una selección ordenada por la frecuencia de su aparición en la literatura: factores legales, demanda de los consumidores, respuesta a los grupos de interés, conseguir una ventaja competitiva, grupos de presión sociales y ambientales y pérdida de reputación.

En el segundo punto, gestión del riesgo y desempeño, los autores analizan cuáles son los factores internos de la SC que dificultan adoptar una gestión sostenible, así como los factores que dan soporte y ayudan a implantar las SSC. Entre las barreras que se encuentran las empresas, los autores mencionan tres: los elevados costes, la complejidad y el esfuerzo para coordinar toda la SC, así como la falta de comunicación en la misma. Por el contrario, para Seuring y MüIler, los factores que ayudan a desarrollar una SSC son: la intercomunicación entre las empresas, la adopción de sistemas de gestión (ISO I 4000, SA8000, etc.), la realización de seguimiento, evaluaciones, presentación de informes e imposición de sanciones, la formación de empleados y proveedores para la realización de las compras, y la integración en la política de la empresa.

En el último punto, la gestión para conseguir productos sostenibles, los autores proponen a las compañías adoptar el método de la evaluación del ciclo de vida para especificar las características de los productos. En este punto se vuelve a hacer mención a la importancia que tiene la colaboración entre todos los agentes de la SC y el control que debe realizar la empresa principal para que la cadena completa cum- pla las características de calidad y desempeño del proceso productivo definidas.

Finalmente, los autores señalan como temas en los que seguir profundizando, entre otros: la definición de un amplio conjunto de objetivos de rendimiento en los que se tenga en cuenta la dimensión medioambiental y social y la necesidad de aumentar la cooperación entre todos los socios para la SSCM.

\subsection{El modelo de Pagell y Wu}

Pagell y Wu (2009) proponen un modelo basado en las prácticas que debe realizar una empresa para conseguir una SSC. El modelo fue desarrollado después de estudiar cuáles eran las prácticas que realizaban I 0 empresas líderes en sostenibilidad dentro de su sector de actividad.

Para los autores, los gestores de las SC deben: identificar quién está presente en la cadena y cuál es el objetivo de la misma, y asegurar que es efectiva y eficiente $y$, por último, modificar la comunicación e introducir los problemas medioambientales y sociales como una manera de conseguir beneficios económicos para la empresa. Además, proponen que se implique a los empleados, para lo cual, es necesario proporcionarles la información necesaria para que puedan pensar sobre la sostenibilidad y compatibilizarla con los objetivos empresariales.

En la Figura 3 se resume el modelo propuesto por Pagell y Wu, donde es importante destacar como elemento diferenciador respecto al resto de modelos

Figura 3

Un modelo de prácticas de SSCM. Fuente: Pagell y Wu (2009)

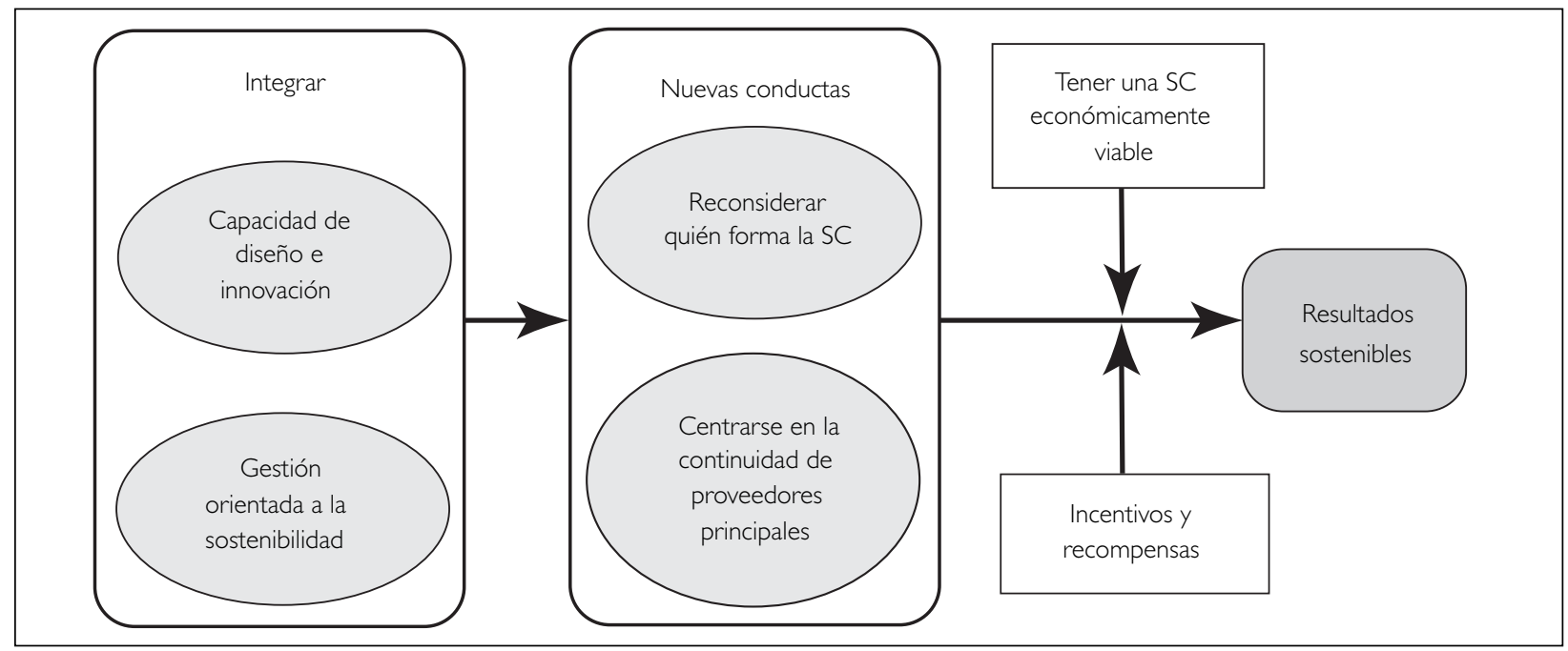


el que se considere que la sostenibilidad estará integrada en la organización cuando tenga, tanto la gestión orientada a la sostenibilidad, como la capacidad de innovación. Respecto a lo demás, hay bastante coincidencia en cuanto a la importancia de la comunicación y la participación de todos los agentes de la SC (proveedores, empleados y otros grupos de interés) para lograr desarrollar una verdadera SSC.

En sus conclusiones, Pagell y Wu (2009) critican que los estudios existentes realizan el análisis de la SSCM bajo el mismo enfoque («iqué deben hacer las empresas?»). En cambio, su estudio presenta un enfoque diferente: exploran directamente lo que un conjunto de empresas sostenibles hacen de forma única y que, por lo tanto, pueden comunicar a otros para que traten de seguir el mismo camino. Para futuras investigaciones, los autores proponen extender el estudio y examinar las prácticas de las organizaciones más típicas, no solo las de las compañías líderes en sostenibilidad de cada sector; además, proponen examinar operaciones, diseño logístico y otras actividades con el fin de ver si existen otros comportamientos importantes que deban ser adoptados por las empresas de la SC, líderes en sostenibilidad, que hayan podido ser omitidas en su estudio.

\section{Modelo de aplicación/despliegue de la CSR en la SCM}

Tras la breve revisión de los tres modelos propuestos para lograr una SSCM, el objetivo de este trabajo es definir un enfoque que recoja la mayor parte de la visión compartida de estos modelos y los complemente con una perspectiva más aplicada o de despliegue de los conceptos planteados hacia toda la organización. De este modo, se pretende que las empresas puedan aprovechar sus actuales planteamientos estratégicos, de gestión y operativos y reenfocarlos o alinearlos con la CSR.

Se comparte, lógicamente, la base de partida de los tres modelos en lo que se refiere a la triple dimensión con la que debe plantearse la sostenibilidad de la SC: económica, social y medioambiental. Se comparte también el marco conceptual acerca de los facilitadores o incentivos/recompensas que entran en juego para promover el desarrollo de una SSCM. Lo que se considera necesario es el incorporar al modelo los conceptos de estrategia, organización, diseño y gestión, que forman parte del desarrollo común de la actividad de las empresas, y que pueden ayudar a definir con mayor aplicabilidad muchas de las prácticas que se plantean de manera más general en los modelos analizados.
Por otra parte, si bien los modelos analizados plantean la necesidad de que exista colaboración entre los diferentes agentes de la SC y la necesidad de hacer que fluya la comunicación entre los mismos y, además, concretamente Pagell y Wu, promueven la implicación de los empleados, se considera que estos modelos no desarrollan suficientemente los aspectos organizativos que deben dar soporte a la SSCM.

En la Figura 4 se muestran los tres elementos que configuran el modelo propuesto en este trabajo: la integración de la estrategia de la SCM en la estrategia CSR de la empresa, la estructura organizativa de soporte al desarrollo de esta estrategia integrada y las herramientas necesarias para desplegar la estrategia a lo largo de toda la estructura organizativa de la SCM.

En primer lugar, en lo que respecta a relación de la estrategia definida para la SCM dentro de cada organización y el papel que juegue la CSR en la estrategia de la empresa, se valora la visión de Porter y Kramer (2006) en su propuesta de evolucionar de un planteamiento reactivo de CSR que supone, en lo que respecta a la cadena de valor, tratar de mitigar el daño causado por las actividades de la cadena de valor, a un planteamiento estratégico de CSR, buscando trasformar las actividades de la cadena de valor para beneficio de la sociedad. En este sentido, la estrategia de la SCM debe integrarse dentro del marco más amplio de la CSR estratégica, contribuyendo al enfoque proactivo o de mejora continua con las que deben transformarse las actividades de la cadena de valor. Se considera que los modelos como Lean Management, EFQM, Total Quality Management, etc., que han venido aplicando las empresas en la últimas décadas en la búsqueda de ventajas competitivas a través de la orientación al cliente y la eficiencia en la reducción de costes, son perfectamente válidos para contribuir a la transformación proactiva en la SCM, de manera que incorpore a la idea de eficiencia, la perspectiva de la sostenibilidad, no sólo la del coste.

Por otra parte, se estima también acertada la perspectiva de Pagell y Wu (2009), al plantear que no debe descartarse el superar la aportación del Lean/TQM como enfoques de mejora continua o progresiva, integrando a la gestión para la sostenibilidad una capacidad de innovación que permita lograr, cuando sea necesario, los cambios radicales en la organización que ayuden a avanzar hacia una verdadera sostenibilidad. 
Figura 4

Modelo de aplicación de la CSR en la SCM. Fuente: elaboración propia

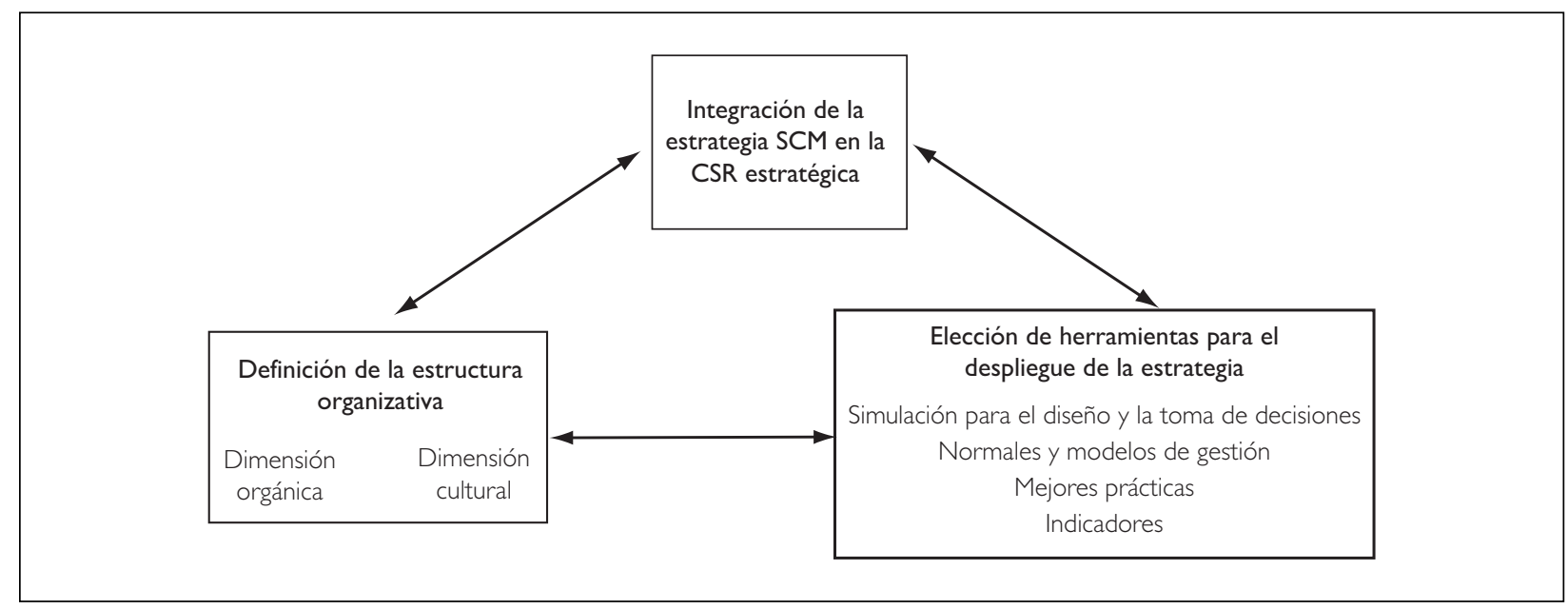

El segundo de los elementos del modelo propuesto es la definición de la estructura organizativa para la SSCM. Como se ha comentado, este aspecto no se define de manera explícita en los modelos anteriormente analizados y, sin embargo, en todos ellos se hace referencia a la necesidad de lograr la cooperación entre todos los agentes de la cadena, la comunicación y la implicación. Entendemos que todo ello debe desarrollarse a todos los niveles de interacción entre los agentes, haciendo que la gestión de flujos de material, información y capital esté orientada a lograr objetivos en las tres dimensiones del desarrollo sostenible. Por otra parte, el logro de estos objetivos no responde sólo al cumplimento de una responsabilidad profesional, sino que existe un componente ético y de implicación con lo que ello conlleva y para lo que algunas personas pueden mostrar una predisposición natural y otras, en cambio, pueden necesitar un estímulo a través de la formación y la sensibilización.

Desde este punto de vista, la definición de la estructura organizativa para la SSCM requiere el planteamiento de dos dimensiones. En primer lugar, una dimensión que se ha denominado orgánica y que hace referencia a la propia articulación de la estructura organizativa para la aplicación de la CSR en la SCM. En concreto, se propone una estructura basada en Grupos Estructurados de Participación, multijerárquicos, multidepartamentales y multiempresa. Las razones que justifican esta propuesta son tres. En primer lugar, la participación debe entenderse como una práctica de la CSR dentro de la perspectiva social con la que dar respuesta a los intereses de los stakeholders internos, concretamente, los emplea- dos de las organizaciones. Por otra parte, dado que una de las principales barreras para lograr una verdadera gestión integrada de la SC es la falta de coordinación, en lo que coinciden Carter y Jennings (2002) al señalar la falta de coordinación de objetivos y actividades entre las áreas funcionales internas (integración de la logística interna) y entre los diferentes agentes de la SC como principales obstáculos en la LSR, esta estructura de grupos de participación favorece la coordinación en diferentes niveles de decisión de los procesos y actividades logísticas. Por último, creemos que la participación «dirigida» o alineada con los objetivos estratégicos puede contribuir a la transformación de las actividades en beneficio de la sociedad.

En cuanto a la dimensión cultural, se coincide con Carter y Rogers (2008) en que la cultura de las organizaciones tiene que ser uno de los pilares que deben dar soporte a la triple cuenta y que debe estar profundamente integrada, reflejar una ciudadanía corporativa y basarse en la ética y en unos valores. Dentro de esta dimensión cultural, como se ha señalado anteriormente, se plantea la formación y sensibilización de las personas como medio de crear/enriquecer la cultura organizativa hacia la CSR a través de los valores personales de todos los miembros de la organización.

Por último, los aspectos de diseño y la gestión de la SC a los que se enfrentan las empresas, las relaciones entre los diferentes agentes, la conducta y transparencia en el desarrollo de las actividades o la valoración del desempeño alcanzado en todas las actividades, son considerados en los anteriores mo- 
delos estudiados. Estos aspectos, también se integran en el modelo propuesto en este trabajo como tercer elemento de configuración, bajo el concepto de herramientas necesarias para el despliegue de la estrategia a lo largo de toda la SC.

En primer lugar, consideramos estratégica la decisión de elegir los «socios» (en especial, los principales proveedores) que formarán parte de la SC y, como señalan Pagell y Wu (2009), la definición de nuevas conductas para la sostenibilidad. El diseño de la SC juega asimismo un papel fundamental en la contribución a la triple cuenta de la SCM; basta considerar las implicaciones que la red de infraestructuras (fábricas, almacenes, medios de transporte, etc.) de la SC tienen en el ámbito social (por ejemplo, creación de empleo local), medioambiental (por ejemplo, consumo de recursos naturales como materias primas, combustible,...) y económico (por ejemplo, en la propia rentabilidad de la actividades logísticas). A este respecto, se considera que el uso de las técnicas de simulación, por ejemplo, puede suponer una ayuda muy eficiente a la toma de decisiones en las que entran en juego múltiples variables y una gran incertidumbre.

En segundo lugar, a nivel táctico, la implantación de sistemas de gestión ISO 900 I, I 400 I y OHSAS I 800 I ha permitido crear en las empresas una dinámica de gestión orientada a los procesos y a la mejora continua, además de crear un marco común de referencia sobre la gestión de las empresas en materia de calidad, medioambiente y seguridad laboral que nos acercan a los planteamientos de la CSR. De manera similar, la necesidad de crear un marco de referencia para la implantación de la CSR en las empresas ha originado la aparición de diferentes guías, estándares y modelos, algunas de las cuales cubren todos los ámbitos de definición de la CSR (económico, social y medioambiental), si bien otros sólo se centran en alguno de estos aspectos. A modo de ejemplo se puede citar AA I 000, SA8000 o ISO 26000.

A pesar de que la proliferación de guías y estándares puede crear confusión en las empresas a la hora de definir la manera en la que deben integrar la gestión CSR internamente y a los largo de la SCM, confiamos en que a medida que las iniciativas por parte de las empresas sean más abundantes y consistentes, se produzca una convergencia hacia modelos o estándares universales.

Por último, las mejores prácticas que favorezcan la eficiencia y la sostenibilidad (de aplicación desde el ámbito estratégico, pasando por el táctico y, final- mente, por el operativo) son las terceras herramientas que consideramos necesarias para el despliegue de la CSR en toda la organización y a lo largo de la SC. Si bien son ya diversas las mejores prácticas que se han adoptado para la mejora de la gestión en la SC (desde una perspectiva de ahorro de costes) y que, además, contribuyen a la mejora de aspectos medioambientales y/o sociales, diferentes autores, entre los que se incluyen los de los tres modelos estudiados, señalan la necesidad de investigar más profundamente para detectar nuevas y mejores prácticas que pueden adoptar las empresas para garantizar la sostenibilidad de la SCM.

Finalmente, el desarrollo de un sistema de indicadores, apropiado a cada uno de los niveles de gestión y adecuado para cada uno de los procesos y actividades logísticas, permitirá medir el desempeño alcanzado y definir las acciones correctoras y preventivas que contribuyan a la mejora continua.

\section{Conclusiones y siguientes desarrollos}

En este trabajo se han recogido las principales revisiones llevadas a cabo en lo relativo a la literatura general de la CSR y a la literatura sobre CSR que incorpora el área funcional logística (o algunas de sus actividades) dentro de su estudio. En lo que respecta a esto último, se observa que el tratamiento que en la literatura se ha venido dando a las implicaciones de la CSR en la SCM es limitado y relativamente reciente, respondiendo, en la mayoría de los casos, al estudio de manera independiente de ámbitos concretos dentro de la cadena.

Sin embargo, en los últimos años, el concepto de sostenibilidad aplicado a la SCM, apoyado a su vez en el concepto de la «triple cuenta» desarrollado por Elkington $(1998,2004)$, ha permitido crear un contexto sobre el que poder definir teorías y plantear estudios empíricos en un campo todavía muy poco explorado.

Precisamente, este trabajo pretende ser una contribución teórica a este campo, al complementar la visión ofrecida por los tres modelos existentes que se han identificado en la literatura sobre SSCM. Con el modelo propuesto se busca ofrecer una perspectiva más aplicada o de despliegue de los conceptos planteados en los tres modelos analizados hacia toda la organización, de manera que las empresas puedan aprovechar sus actuales planteamientos estratégicos, de gestión y operativos y reenfocarlos o alinearlos con la CSR. 
Dentro del modelo propuesto, destacamos especialmente la incorporación de manera explícita de los aspectos organizativos que deben ser tenidos en cuenta para implicar a todos los agentes de la SC en la sostenibilidad de la gestión, logrando a su vez, actuaciones coordinadas a los largo de toda la cadena. En este sentido, se considera que una organización basada en sistemas de participación estructurados multijerárquicos, multidepartamentales y multiempresa constituyen una solución muy adecuada para conseguir tales objetivos.

Los trabajos analizados en la literatura señalan la necesidad de profundizar todavía más en los planteamientos teóricos de esta nueva realidad empresarial. Descubrir las motivaciones de las empresas para introducirse en la aplicación de la CSR en la SCM o, lo que es lo mismo, transformar la SCM en sostenible, conocer las prácticas desarrolladas por la empresas líderes o avanzadas en la sostenibilidad de sus SC y descubrir prácticas innovadoras, estudiar la manera de introducir a la Pymes dentro de este enfoque o definir los instrumentos para medir el rendimiento que se logra a través de la sostenibilidad, son sólo algunas de las cuestiones que se plantean en la literatura como necesarias para completar el marco teórico actual sobre SSCM.

Como líneas futuras en el desarrollo de este modelo, los autores proponen definir de manera más detallada cada uno de los elementos de este marco de cara a poder profundizar en ellos y otorgar, así, más aplicabilidad al modelo. Tras ello, será oportuno pasar de la fase conceptual a la fase empírica, con objeto de validar el modelo en el contexto empresarial.

\section{Bibliografía}

BOWEN, H. (1953). Social responsibilities of the businessman, New York: Ed Harper.

CARROLL, A.B. (1999). «Corporate social responsibility. Evolution of a definitional construct», Business \& Society, 38, pp 268-295.

CARROLL, A.B. (1979). «A three-dimensional conceptual model of corporate performance», Academy of Management Review, 4, pp 497-505

CARTER, C.RY ROGERS, D.S. (2008). «A framework of sustainable supply chain management: moving toward new theory», International Journal of Physical Distribution \& Logistics Management, 38 (5), pp 360-387.

CARTER, C.R.Y JENNINGS, M.M. (2002). «Logistics social responsibility: an integrative framework», Journal of Business Logistics, 23 (I), pp |45- I 80.
CARTER, C.R. (2000a). «Ethical issues in international buyer-supplier relationships: a dyadic examination», Journal of Operations Management, I 8 (2), pp | 9 |-208.

CARTER, C. R. (2000b). «Precursors of unethical behavior in global procurement», Journal of Supply Chain Management, 36 (I), pp 45-57.

CARTER, C.R.; AUSKALNIS, R. Y KETCHUM, C. (1999). «Purchasing from minority business enterprises: a crossindustry comparison of best practices», Journal of Supply Chain Management, 35 ( I), pp 28-32.

COOPER, M.C.Y GILLYARD, A. (2000). «2000 career patterns of women in logistics», Annual Conference Proceedings of the Council of Logistics Management, OakBrook, IL: Ed Council of Logistics Management, pp 75-97.

ELKINGTON, J. (2004). «Enter the triple bottom line», in HENRIQUES, A. Y RICHARDSON, J. (Eds). The triple bottom line: Does it all add up?, London: Ed Earthscan, pp |- 16 .

ELKINGTON, J. ( 1998). Cannibals with forks:The triple bottom line of the 2 I st century, Stoney Creek, CT.: Ed New Society Publishers.

ELTANTAWY, R.A.; FOX, G.L. Y GIUNIPERO, L. (2009). «Supply management ethics responsibility: reputation and performance impacts», Supply Chain Management: an International Journal, 14 (2), pp 99- 108.

EMMELHAINZ, M.A.Y ADAMS, R.J. (1999). «The apparel industry response to 'sweatshop' concerns: a review and analysis of codes of conduct», Journal of Supply Chain Management, 35 (3), pp 5 । -57.

HANDFIELD, R., SROUFE, S. Y WALTON, S. (2005). «Integrating environmental management and supply chain strategies», Business Strategy and the Environment, 14 ( I), pp I-19.

HANDFIELD, R.B.; WALTON, S.V.; SEEGERS, L.K. Y MELNYK, S.A. (1997). «Green value chain practices in the furniture industry», Journal of Operations Management, I5 (3), pp 293-3I 5.

HART, S. (1997). «Beyond greening: strategies for a sustainable world», Harvard Business Review, (75), pp 6676.

LEE, M.P. (2008). «A review of the theories of corporate social responsibility: its evolutionary path and the road ahead», International Journal of Management Reviews, 10 ( I), pp 53-73.

McKINNON,A.C.; STIRLING, I.Y KIRKHOPE, J. (1993). «Improving the fuel efficiency of road freight operations», International Journal of Physical Distribution and Logistics Management, 23 (9), pp 3-II.

MOLLENKOPF, D; STOLZE, H.; TATE, W.L. Y UELTSCHY, M. (20I0). «Green, lean, and global supply chains», In- 
ternational Journal of Physical Distribution and Logistics Management, 40 ( I-2), pp |4-4I.

PORTER, M.E.Y KRAMER, M.R. (2006). «Strategy and society», Harvard Business Review, (84), pp 78-92.

PORTER, M.E.Y KRAMER, M.R. (2002). «The competitive advantage of corporate philanthropy», Harvard Business Review, (80), pp 56-68.

PORTER, M.E.Y LINDE, C.V.D. (1995). «Green and competitive: ending the stalemate», Harvard Business Review, 73 (5), pp I 20- I 35.

PORTER, M.E. (1 980). Competitive strategy, New York: Ed Free Press.

SRIVASTAVA, S.K. (2007). «Green supply-chain management: a state-of the-art literature review», International Journal of Management Reviews, 9 ( I ), pp 53-80.

RANDALL, S. ( 1 998). «Gaining an ergonomic edge. Modem materials handling», 53 ( I0), pp 44-46.

SARKIS, J. (2003). «A strategic decision framework for green supply chain management», Journal of Cleaner Production, II, pp 397-409.

SEURING, S.Y MÜLLER, M. (2008). «From a literature review to a conceptual framework for sustainable supply chain management», Journal of Cleaner Production, 16, pp 1699-1710.
SHARP, J. M.; NOVACK, R.A. Y ANDERSON, M.A. (199|). «Purchasing hazardous waste transportation service: federal legal considerations», Transportation Journal, 3 । (2), pp 4- 4.

STOCK, J.R. (1998). Development and implementation of reverse logistics programs, Oak Brook, IL: Ed Council of Logistics Management.

STOCK, J.R. (1992). Reverse logistics, Oak Brook, IL: Ed Council of Logistics Management.

VACHON, S. Y KLASSEN, R.D. (2006). «Extending green practices across the supply chain», International Journal of Operations \& Production Management, 26 (7), pp 795-821.

WARTICK, S.L.Y COCHRAN, P.L. (1985). «The evolution of the corporate social performance model», Academy of Management Review, I0, pp 758-769.

WEENER, E.F.Y WHEELER, P.B. (1992). «Key elements of accident avoidance», Logistics and Transportation Review, 28 ( I), pp 49-60.

WOOD, D.J.Y JONES, R.E. (1995). «Stakeholder mismatching: a theoretical problem in empirical research on corporate social performance», International Journal of Organizational Analysis, 3, pp 229-267.

WORLD COMMISSION ON ENVIRONMENT AND DEVELOPMENT (1987). Our Common Future, New York: Ed Oxford University Press. 